\title{
Discrete Breathers in One-Dimensional Diatomic Granular Crystals
}

\author{
N. Boechler, ${ }^{1}$ G. Theocharis, ${ }^{1,2}$ S. Job, ${ }^{3,1}$ P. G. Kevrekidis, ${ }^{2}$ Mason A. Porter, ${ }^{4}$ and C. Daraio ${ }^{1}$ \\ ${ }^{1}$ Graduate Aerospace Laboratories (GALCIT), California Institute of Technology, Pasadena, California 91125, USA \\ ${ }^{2}$ Department of Mathematics and Statistics, University of Massachusetts, Amherst, Massachusetts 01003-4515, USA \\ ${ }^{3}$ Supmeca, 3 rue Fernand Hainaut, 93407 Saint-Ouen, France \\ ${ }^{4}$ Mathematical Institute, University of Oxford, OX1 3LB, United Kingdom
}

(Received 13 November 2009; published 18 June 2010)

\begin{abstract}
We report the experimental observation of modulational instability and discrete breathers in a onedimensional diatomic granular crystal composed of compressed elastic beads that interact via Hertzian contact. We first characterize their effective linear spectrum both theoretically and experimentally. We then illustrate theoretically and numerically the modulational instability of the lower edge of the optical band. This leads to the dynamical formation of long-lived breather structures, whose families of solutions we compute throughout the linear spectral gap. Finally, we experimentally observe the manifestation of the modulational instability and the resulting generation of localized breathing modes with quantitative characteristics that agree with our numerical results.
\end{abstract}

DOI: 10.1103/PhysRevLett.104.244302

Introduction.-Intrinsic localized modes, or discrete breathers (DBs), have been a central theme in nonlinear investigations during the past two decades [1,2]. Their original theoretical proposal in settings such as anharmonic lattices [3] and the rigorous proof of their existence under fairly general conditions [4] motivated studies of such modes in a diverse host of applications, including chargetransfer solids [5], antiferromagnets [6], superconducting Josephson junctions [7], photonic crystals [8], biopolymers [9], micromechanical cantilever arrays [10], and more.

Granular crystals consist of closely packed ensembles of elastically interacting particles. Recent interest has arisen from their tunable dynamic response encompassing linear, weakly nonlinear, and strongly nonlinear regimes $[11,12]$. Such flexibility, arising from the nonlinear contact interaction between particles, makes them ideal not only as toy models for probing the physics of granular materials but also for the implementation of engineering applications, including shock and energy absorbing layers [13], actuating devices [14], and sound scramblers [15]. Only recently have nonlinear localized modes begun to be explored in granular crystals. Previous studies have focused on metastable breathers in an acoustic vacuum [16], the observation of localized oscillations near a defect $[17,18]$, and onedimensional (1D) diatomic crystals restricted to linear dynamics due to welded sphere contacts [19]. Understanding and controlling localization in granular crystals might lead to new energy-harvesting or filtering devices.

In this Letter, we use experiments, theory, and numerical simulations to investigate the existence, stability, and dynamics of DBs in a compressed 1D diatomic granular crystal. The DB consists of particles that oscillate with a frequency in the forbidden band (i.e., the gap) of the linear spectrum and with an amplitude which decreases exponentially from the central particle. We first detail our experimental setup and theoretical model. We then analyze the
PACS numbers: 45.50.Dd, 05.45.Yv, 45.70.-n, 46.40.Cd

system's dynamics in the linear regime, show how a modulational instability (MI) generates DBs in the weakly nonlinear regime, and finally provide experimental evidence of their existence.

Experimental setup.-We assemble a 1D diatomic granular crystal by alternating aluminum spheres (6061T6 type, radius $R_{a}=9.525 \mathrm{~mm}$, mass $m_{a}=9.75 \mathrm{~g}$, elastic modulus $E_{a}=73.5 \mathrm{GPa}$, Poisson ratio $\left.\nu_{a}=0.33\right)$ and stainless steel spheres (316 type, $R_{b}=R_{a}, m_{b}=28.84 \mathrm{~g}$, $\left.E_{b}=193 \mathrm{GPa}, \nu_{b}=0.3\right)$. The reported values of $E_{a, b}$ and $\nu_{a, b}$, are standard specifications [20]; we discuss the precise characterization of the effective elastic properties of our system below. We hold the spheres in place using four polycarbonate restraining bars and guide plates. At one end of the crystal, we apply a precompressive force using a lever-mass system. We drive the crystal dynamically with a piezoelectric actuator that we fit on a steel plate clamped on a steel bracket (called the "wall" in Fig. 1). We visualize the evolution of the force-time history of the propagating excitations using calibrated, periodically placed piezo sensors that we embed inside selected particles (preserving the inertia and the bulk stiffness of the original bead $[12,15])$. We measure the static load using a calibrated strain gauge cell that we place in contact with the lever arm and with the last bead of the crystal.

Theoretical model.-We model a 1D diatomic crystal of $\mathrm{N}$ spheres as a chain of nonlinear oscillators [11]:

$$
m_{i} \ddot{u}_{i}=A\left[\delta_{0}+u_{i-1}-u_{i}\right]_{+}^{p}-A\left[\delta_{0}+u_{i}-u_{i+1}\right]_{+}^{p},
$$

where $[Y]_{+}$denotes the positive part of $Y, u_{i}$ is the displacement of the $i$ th sphere (where $i \in\{1, \ldots, N\}$ ) around the static equilibrium, the masses are $m_{\text {odd }}=m_{a}$ and $m_{\text {even }}=m_{b}$, and the coefficient $A$ depends on the exponent $p$ and the geometry or material properties of adjacent beads. The exponent $p=3 / 2$ yields the Hertz poten- 


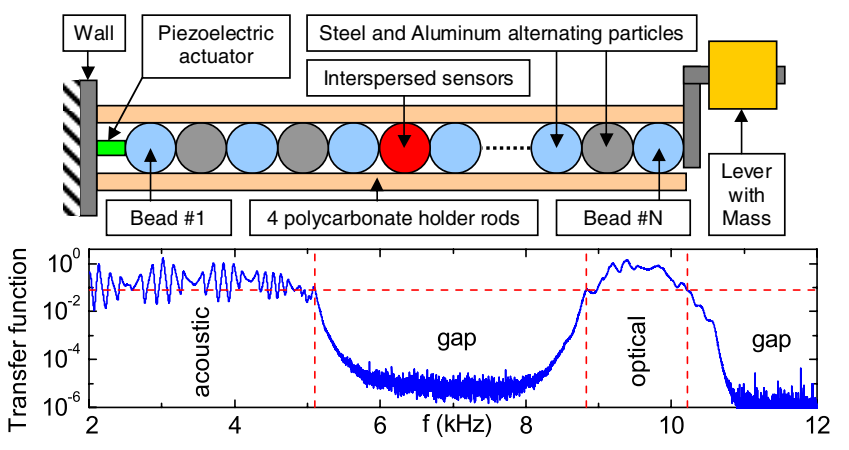

FIG. 1 (color online). Top panel: Experimental setup. Bottom panel: Experimental linear spectrum of the 81-bead steelaluminum diatomic crystal. The horizontal line designates half of the low-frequency mean value, and vertical lines indicate the $f_{n}^{\mathrm{exp}}$ cutoff frequencies given in Table I.

tial law between adjacent spheres [21]. In this case, $A=\left\{\frac{3}{4}\left[\left(1-\nu_{a}^{2}\right) / E_{a}\right]+\frac{3}{4}\left[\left(1-\nu_{b}^{2}\right) / E_{b}\right]\right\}^{-1}\left(\frac{1}{R_{a}}+\frac{1}{R_{b}}\right)^{-1 / 2}$ and one obtains a static overlap of $\delta_{0}=\left(F_{0} / A\right)^{2 / 3}$ under a static load $F_{0}$ [11,12]. We compute the linear dispersion relation of our system from the linearization of Eq. (1). For diatomic crystals, this curve contains two branches (acoustic and optical) [22]. At the edge of the first Brillouin zone-i.e., at $k=\frac{\pi}{2 \alpha}$, where $\alpha=R_{a}+R_{b}-\delta_{0}$ is the equilibrium distance between two adjacent beads-the linear spectrum possesses a gap between the upper cutoff frequency $\omega_{1}=\sqrt{2 K_{2} / M}$ of the acoustic branch and the lower cutoff frequency $\omega_{2}=\sqrt{2 K_{2} / m}$ of the optical branch. The linear stiffness is $K_{2}=\frac{3}{2} A^{2 / 3} F_{0}^{1 / 3}$, and $M=$ $\max \left\{m_{a}, m_{b}\right\}$ and $m=\min \left\{m_{a}, m_{b}\right\}$. The upper cutoff frequency of the optical band is $\omega_{3}=\sqrt{2 K_{2}(1 / m+1 / M)}$. In Table I, we summarize $K_{2}, A$, and the three cutoff frequencies, which we estimate using standard specifications [20] and compute using a static load of $F_{0}=20 \mathrm{~N}$.

Linear spectrum.-We experimentally characterize the linear spectrum of a diatomic crystal [23] $(N=81$ and $F_{0}=20 \mathrm{~N}$ ) by applying low-amplitude (approximately $10 \mathrm{mN}$ peak), broadband frequency $(2-18 \mathrm{kHz})$, and uniform noise for $800 \mathrm{~ms}$. We measure the dynamic force using a sensor located inside the fourteenth particle, and derive the input force from the driving voltage multiplied by the actuator sensitivity. We then compute the power spectral density (PSD) of the force sensor, normalize it to the PSD of the driving force, and average the ratio over 8 acquisitions to obtain the transfer function shown in Fig. 1. This spectrum clearly shows forbidden bands (i.e., gaps) and pass bands bounded by cutoff frequencies. These frequencies match half of the transfer function's lowfrequency level, which we compute as the mean level in the $2-4 \mathrm{kHz}$ range. We summarize these frequencies in Table I. Matching these frequencies to the theoretical formulas above provides an opportunity to probe the beads' effective parameters $K_{2}$ and $A$ shown in Table I (error bars indicate the standard deviations from the three frequency measurements). All cutoff frequencies show a systematic upshift of about 9\% compared to the predictions from standard specifications. We have identified four possible explanations: (i) the uncertainty in the standard values of material parameters [20]; (ii) non-Hookean elastic dynamics might lead to a slight shift in the nonlinear exponent $p$ and accordingly a large deviation in the coefficient A [21]; (iii) imperfect surface smoothness might induce fluctuations in $p$ and hence in $A$ [24]; and (iv) dissipative mechanisms, such as viscoelasticity and solid friction, can induce stiffening of the interaction potential between particles $[12,25]$.

Modulational Instability and DBs.-We now consider the weakly nonlinear dynamics of the granular crystal. If the displacements have small amplitudes relative to those due to precompression, we can take a power series expansion of the forces (up to quartic displacement terms) to yield the $K_{2}-K_{3}-K_{4}$ model:

$$
m_{i} \ddot{u}_{i}=\sum_{k=2}^{4} K_{k}\left[\left(u_{i+1}-u_{i}\right)^{k-1}-\left(u_{i}-u_{i-1}\right)^{k-1}\right]
$$

where $K_{3}=-\frac{3}{8} A^{4 / 3} F_{0}^{-1 / 3}$ and $K_{4}=\frac{3}{48} A^{2} F_{0}^{-1}$. Equation (2) constitutes a diatomic variant of the Fermi-Pasta-Ulam nonlinear oscillator chain [26]. Because $K_{3}^{2} / K_{2} K_{4}>\frac{3}{4}$, we expect the lower optical cutoff mode, for which the light masses oscillate out of phase at frequency $f_{2}^{\exp }$ and the heavy masses are at rest, to be subject to MI [27], which is a principal mechanism for energy localization in nonlinear lattices [28]. In order to verify this prediction, we numerically solve Eq. (1) using $A_{\exp }$ (see Table I) and the lower optical cutoff mode as the initial condition. To trigger the MI, we choose an initial oscillation amplitude of the light masses that corresponds to an $11.25 \mathrm{~N}$ (i.e., $0.5625 F_{0}$ ) dynamic peak force. As shown in Fig. 2(a), this method allows us to observe the MI and the resulting generation of a localized mode, after $t \simeq 8 \mathrm{~ms}$, with frequency $f_{b}=$ $7.95 \mathrm{kHz}$ in the gap. In Fig. 2(a2), one can observe an exponential growth, which is characteristic of MI, around $t \simeq 5 \mathrm{~ms}$. A more convenient way to excite the lower optical cutoff mode is to drive the chain at one end with a sine wave at the lower optical cutoff frequency, $f_{\text {act }}=$ $f_{2}^{\text {exp }}$. In Fig. 2(b1), we show an example of the spatiotemporal evolution of the forces when the chain is driven during $30 \mathrm{~ms}$ (the amplitude of the first bead's displacement is about $0.061 \delta_{0}$ ). In this example, the maximum dynamic force acting on the beads over the first 10 cycles

TABLE I. Predicted (from standard specifications [20]) versus measured cutoff frequencies, linear stiffness $K_{2}$, and coefficient $A$ under a static precompression of $F_{0}=20 \mathrm{~N}$.

\begin{tabular}{lccccc}
\hline \hline & $f_{1}[\mathrm{kHz}]$ & $f_{2}[\mathrm{kHz}]$ & $f_{3}[\mathrm{kHz}]$ & $K_{2}[\mathrm{~N} / \mu \mathrm{m}]$ & $A\left[N / \mu \mathrm{m}^{3 / 2}\right]$ \\
\hline Theory & 4.71 & 8.10 & 9.37 & 12.63 & 5.46 \\
Experiment & 5.11 & 8.83 & 10.22 & $14.95 \pm 0.10$ & $7.04 \pm 0.07$ \\
Difference & $+8.5 \%$ & $+9.0 \%$ & $+9.1 \%$ & $+18.4 \%$ & $+28.8 \%$ \\
\hline \hline
\end{tabular}


of the excitation is about $6.5 \mathrm{~N} \simeq 0.325 F_{0}$. We thus anticipate a weakly nonlinear response that is well described by the $K_{2}-K_{3}-K_{4}$ theory. Indeed, during the first $20 \mathrm{~ms}$, the lower optical cutoff mode is established, followed by an MI after $t \simeq 22 \mathrm{~ms}$. The width of the extended lattice wave is decreased, its amplitude is increased, and-as a result of the spontaneous symmetry breaking induced by the instability - a DB is subsequently formed, which for these initial conditions, is localized near bead 37. This nonlinear solution exists even after the actuator is turned off at $t=30 \mathrm{~ms}$. The PSD of the force at particle 36 [see Fig. 2(b2)] reveals the presence of a frequency component in the gap at $f_{b} \simeq 8.14 \mathrm{kHz}<f_{2}^{\text {exp }}$. Numerical simulations show that the final location of the DB depends on the features of the driving signal (amplitude, frequency, and duration). Thus, the exact localized pinning site is not known a priori.

Exact solutions and stability of DBs.-We apply Newton's method (see [2] and references therein) with free boundary conditions to numerically obtain, with high precision, the above dynamically generated DB waveforms as exact time-periodic solutions. We then study their linear stability and frequency dependence (within the spectral gap). Continuing this solution within the gap [i.e., for $\left.f \in\left(f_{1}^{\exp }, f_{2}^{\text {exp }}\right)\right]$ starting from the lower optical cutoff mode allows us to trace the entire family of DB solutions. In Fig. 3(a), we show the maximum dynamic force $\max \left(F_{i}\right)$, which is the experimentally observable parameter of the DB solution, as a function of the DB frequency $f_{b}$. As $f_{b} \rightarrow f_{2}^{\text {exp }}, \max \left(F_{i}\right) \rightarrow 0$ and the DBs broaden and finally merge with the linear lower optical cutoff mode. In the insets of Fig. 3(a), we show examples of these solutions with frequencies $f_{b}=8.35 \mathrm{kHz}$ and $f_{b}=8.75 \mathrm{kHz}$. To
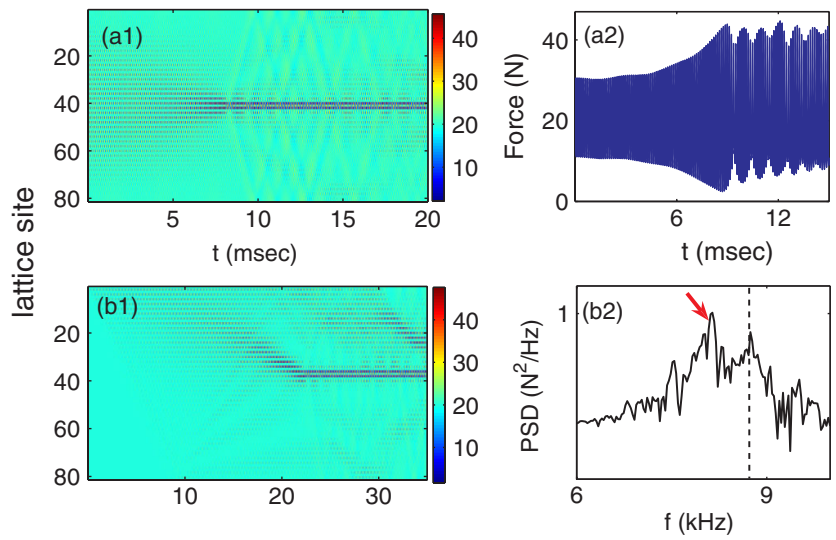

FIG. 2 (color online). (a1) Spatiotemporal evolution of the forces for the simulated manifestation of the MI and DB generation with initial conditions corresponding to the lower optical cutoff mode. (a2) Force versus time for particle 40 for the simulation shown in (a1). (b1) Same as (a1) but for the generation of a DB under conditions relevant to our experimental setup. (b2) PSD of particle 36 for the simulation shown in (b1). The dashed line in (b2) indicates the driving frequency $f_{\text {act }}=f_{2}^{\text {exp }}$, and the arrow indicates the DB frequency $f_{b} \simeq 8.14 \mathrm{kHz}<f_{2}^{\exp }$. examine the stability of the DB solutions, we compute their Floquet multipliers $\lambda_{j}$ [2]. If $\left|\lambda_{j}\right|=1$ for all $j$, then the DB is linearly stable. In Fig. 3(b), we show the stability diagram for the family of DB solutions and the corresponding locations of Floquet multipliers in the complex plane for the DB with $f_{b}=8.63 \mathrm{kHz}$. Strictly speaking, the DB is stable only for $f_{b} \simeq f_{2}^{\text {exp }}$. Otherwise, the DB family exhibits oscillatory instabilities [2,18]. However, the deviations of the unstable eigenvalues from the unit circle are bounded above by 0.08 , and numerical integration of the DBs up to times $100 T$ (where $T$ is their period) reveals their robustness. Importantly, we also find that DB solutions exhibit a strong instability due to a pair of real multipliers when $f_{b} \in(8.45 \mathrm{kHz}, 8.7 \mathrm{kHz})$. As shown in Fig. 3(b), this instability is connected with the turning points of the energy of the DB as a function of its frequency (these occur when $d E / d f_{b}=0$ ). Similar features have also been observed in diatomic Klein-Gordon chains [29].

Experimental observation of DBs.-We excite the 81bead diatomic crystal by driving the actuator with a higheramplitude (relative to the linear-spectrum experiments) $90 \mathrm{~ms}$ sine voltage with frequency close to the lower optical cutoff frequency $f_{2}^{\exp }$. We place force sensors in particles $2,6,10,14,18,22$, and 26. The experimental results in Fig. 4 show the MI onset and subsequent DB formation. Figure 4(a) shows the force versus time at particles 2 (near the actuator) and 14 (close to the DB pinning site), and Fig. 4(b) shows the corresponding PSDs. The peak force amplitude near the actuator is $8.6 \mathrm{~N} \simeq 0.43 F_{0}$ (where $\left.F_{0}=20 \mathrm{~N}\right)$. Figure 4(c) shows the normalized power versus lattice site at both the driving and DB frequencies, before and after the formation of the DB. The normalized power is the PSD at a given frequency divided by the spectral power-i.e., the integral of the PSD over all frequencies. The force at particle 14 shows an exponential increase (at $t \simeq 20 \mathrm{~ms}$ ), which is indicative of the onset of MI. This is followed by the DB formation
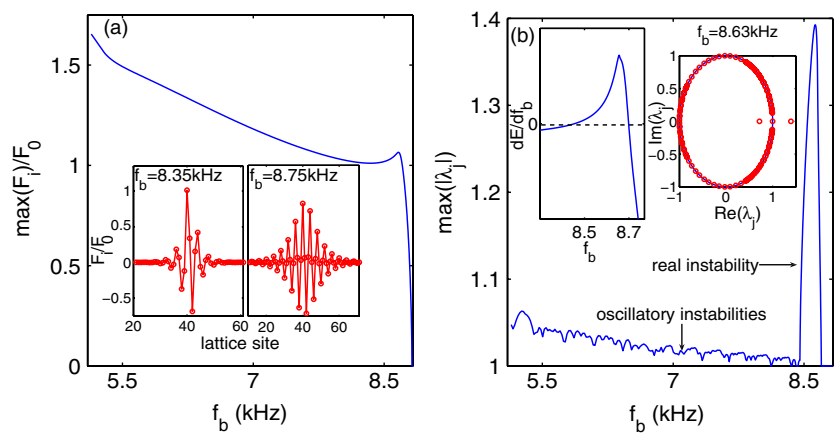

FIG. 3 (color online). Bifurcation diagram of the continuation of the DB solutions. (a) Maximal dynamic force of the wave versus frequency $f_{b}$. The insets show spatial profiles at two values of $f_{b}$. (b) Maximal deviation of Floquet multipliers from the unit circle, which indicates the instability growth rate. The right inset shows a typical multiplier picture, and the left inset shows the connection between the strong (real multiplier) instability and the change in sign of $d E / d f_{b}$. 

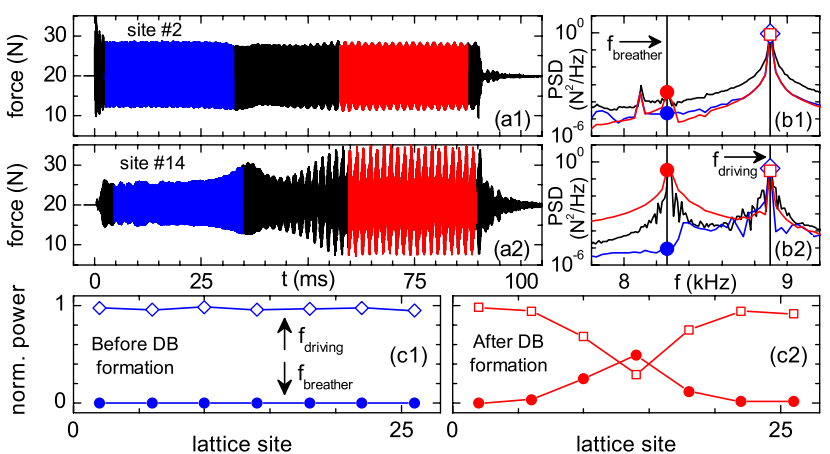

FIG. 4 (color online). Experimental observations of MI and DB at $f_{b}^{\exp } \simeq 8.28 \mathrm{kHz}$, with $f_{1}^{\exp }<f_{b}^{\exp }<f_{2}^{\exp }$, while driving the chain at $8.90 \mathrm{kHz} \simeq f_{2}^{\exp }$ for $90 \mathrm{~ms}$. (a1, a2) Forces versus time and (b1, b2) PSDs at particles 2 and 14. Normalized power versus lattice site at the driving (open symbols) and the DB (filled symbols) frequencies, before (c1) and after (c2) DB formation. Vertical lines in (b) mark the driving and the DB frequency. Dark gray (blue) [light gray (red)] curves in (a, b, c) refer to time regions of $30 \mathrm{~ms}$ before [after] the DB formation, while the black curves refer to the entire signal.

at $t \simeq 55 \mathrm{~ms}$. Both Figs. 4(b) and (c) show the appearance of a frequency component $f_{b}^{\exp } \simeq 8.28 \mathrm{kHz}$ in the gap and localization of the energy over approximately 15 beads around site 14. Before the DB generation, for $t \leq 35 \mathrm{~ms}$, the lattice mostly vibrates at the driving frequency, and the power is uniformly distributed over the lattice [see Fig. 4(c1)]. After the DB formation, for $t \geq 55 \mathrm{~ms}$, part of the energy is pumped from the driving to the DB frequency, as shown in Fig. 4(c2). The decay of the vibrations after the actuator is turned off, which does not occur in the numerical simulations, arises from dissipation $[12,25]$. However, analysis of the PSD after the actuator is turned off indicates that the power at DB frequency is longer-lived than at the driving frequency.

Conclusions.-We have characterized the dynamics of compressed 1D diatomic granular crystals using theory, numerical simulations, and experiments. We found good agreement for the linearized spectrum, explored the mechanism leading to the formation of DBs via MI, and provided clear experimental proof of their existence. Our results provide a first step towards achieving a deeper understanding and classifying intrinsic localized modes in 1D granular crystals and pave the way for their manifestation in 2D and 3D lattices, which might eventually lead to their exploitation in energy-harvesting applications.

We thank R. Carretero-Gonzalez for useful discussions and the NSF for support (Grants No. 0825983, No. 0844540, No. 0806762, and No. 0349023).

[1] S. Aubry, Physica (Amsterdam) 103D, 201 (1997); R. S. MacKay, Physica (Amsterdam) 288A, 174 (2000); D. K. Campbell, S. Flach, and Yu. S. Kivshar, Phys. Today 57 No. 1, 43 (2004).
[2] S. Flach and A. V. Gorbach, Phys. Rep. 467, 1 (2008).

[3] A. J. Sievers and S. Takeno, Phys. Rev. Lett. 61, 970 (1988); J. B. Page, Phys. Rev. B 41, 7835 (1990).

[4] R.S. MacKay and S. Aubry, Nonlinearity 7, 1623 (1994).

[5] B. I. Swanson et al., Phys. Rev. Lett. 82, 3288 (1999).

[6] U. T. Schwarz, L. Q. English, and A. J. Sievers, Phys. Rev. Lett. 83, 223 (1999).

[7] E. Trias, J. J. Mazo, and T. P. Orlando, Phys. Rev. Lett. 84, 741 (2000); P. Binder et al., Phys. Rev. Lett. 84, 745 (2000).

[8] Y.S. Kivshar and G. P. Agrawal, Optical Solitons: From Fibers to Photonic Crystals (Academic Press, San Diego, CA, 2003).

[9] A. Xie et al., Phys. Rev. Lett. 84, 5435 (2000); M. Peyrard, Nonlinearity 17, R1 (2004).

[10] M. Sato, B. E. Hubbard, and A. J. Sievers, Rev. Mod. Phys. 78, 137 (2006).

[11] V.F. Nesterenko, Dynamics of Heterogeneous Materials (Springer-Verlag, New York, NY, 2001); C. Coste, E. Falcon, and S. Fauve, Phys. Rev. E 56, 6104 (1997).

[12] S. Job et al., Phys. Rev. Lett. 94, 178002 (2005).

[13] C. Daraio et al., Phys. Rev. Lett. 96, 058002 (2006); F. Fraternali, M. A. Porter, and C. Daraio, Mech. Adv. Mat. Struct. 17, 1 (2010); F. Melo et al., Phys. Rev. E 73, 041305 (2006).

[14] D. Khatri, C. Daraio, and P. Rizzo, Proc. SPIE Int. Soc. Opt. Eng. 6934, 69340U (2008).

[15] C. Daraio et al., Phys. Rev. E 72, 016603 (2005); V.F. Nesterenko et al., Phys. Rev. Lett. 95, 158702 (2005).

[16] S. Sen and T. R. Mohan, Phys. Rev. E 79, 036603 (2009).

[17] S. Job et al., Phys. Rev. E 80, 025602(R) (2009).

[18] G. Theocharis et al., Phys. Rev. E 80, 066601 (2009).

[19] A. C. Hladky-Hennion, G. Allan, and M. de Billy, J. Appl. Phys. 98, 054909 (2005).

[20] D. R. Christman et al., Measurements of Dynamic Properties of Materials: 6061-T6 Aluminum, Vol. 3 (General Motors Technical Center, Warren, MI, 1971); ASM International, Metals Handbook (ASM International, Materials Park, OH, 1990), 10th ed.

[21] K. L. Johnson, Contact Mechanics (Cambridge University Press, Cambridge, UK, 1985).

[22] C. Kittel, Introduction to Solid State Physics (John Wiley \& Sons, New York, NY, 2005); E. B. Herbold et al., Acta Mech. 205, 85 (2009).

[23] N. Boechler and C. Daraio, Proc. 22nd Biennial Conf. on Mechanical Vibration and Noise (ASME, San Diego, CA, 2009).

[24] C. Coste and B. Gilles, Eur. Phys. J. B 7, 155 (1999).

[25] R. Carretero-Gonzalez et al., Phys. Rev. Lett. 102, 024102 (2009).

[26] S. A. Kiselev, S. R. Bickham, and A. J. Sievers, Phys. Rev. B 48, 13508 (1993); R. Livi, M. Spicci, and R. S. MacKay, Nonlinearity 10, 1421 (1997); P. Maniadis, A. V. Zolotaryuk, and G. P. Tsironis, Phys. Rev. E 67, 046612 (2003).

[27] G. Huang and B. Hu, Phys. Rev. B 57, 5746 (1998).

[28] T. Dauxois and M. Peyrard, Phys. Rev. Lett. 70, 3935 (1993); T. Dauxois et al., Chaos 15, 015110 (2005).

[29] A. V. Gorbach and M. Johansson, Phys. Rev. E 67, 066608 (2003). 